\title{
Potential for the Use of Large Unstructured Data Resources by Public Innovation Support Institutions
}

\author{
Wiesław Cetera ( $\nabla$ w.cetera@uw.edu.pl ) \\ Uniwersytet Warszawski https://orcid.org/0000-0002-5409-4570 \\ Wlodzimierz Gogolek \\ Uniwersytet Warszawski \\ Aleksander Zolnierski \\ Instytut Nauk Ekonomicznych Polskiej Akademii Nauk \\ Dariusz Jaruga \\ Uniwersytet Warszawski
}

\section{Case study}

Keywords: Big Data, information refining, information technologies management, research and development management, research and development support programming, data management, business statistics, innovation

Posted Date: January 28th, 2022

DOI: https://doi.org/10.21203/rs.3.rs-1056779/v1

License: (c) (i) This work is licensed under a Creative Commons Attribution 4.0 International License.

Read Full License 
1 Potential for the use of large unstructured data resources by public innovation support institutions

3

4 Wiesław Cetera Ph. prof. - The Faculty of Political Science and International Studies

5 (Krakowskie Przedmieście 26/28, 00-928 Warsaw)

6 Włodzimierz Gogołek Ph. Prof. - The Faculty of Journalism Information and Book Studies

7 (Krakowskie Przedmieście 26/28, 00-928 Warsaw)

8 Aleksander Żołnierski dr - Institute of Economics of the Polish Academy of Sciences (Staszic

9 Palace, 72 Nowy Świat St. room. 266, 00-330 Warsaw)

Dariusz Jaruga Ph.D. - The Faculty of Political Science and International Studies (Krakowskie

11 Przedmieście 26/28, 00-928 Warsaw)

12

13 Abstract

14 Introduction

15

Effective programming of research and development (R\&D) support, adjusted to the actual potential of beneficiaries, requires the use of modern analytical tools. Efficient R\&D support system requires up-to-date data on technological trends, ongoing (and

18 planning) research, market needs and developing innovation

\section{Case description}

The article presents the potential of one tool that can be applied in public support

21 institutions. Methods of identifying and diagnosing technology trends are presented.

\section{Discussion and Evaluation}

23 The use of methods for refining information from unstructured data is one of the most

24 effective methods for identifying and diagnosing areas requiring support from public

25 funds. Public institutions, including public institutions supporting R\&D and innovation 
processes, can apply tools that allow an increase in the quality of offered support programmes, but also beneficial for the excellence of strategic resources management within the institution itself.

\section{Conclusions}

The described tools and methods are already directly applicable in many areas related to the support of R\&D activity worldwide. The article presents a solution that effectively enables the management of more precise programmes supporting innovative activities.

\section{Keywords}

Big Data, information refining, information technologies management, research and development management, research and development support programming, data management, business statistics, innovation

\section{JEL codes}

C810, H110, O320

\section{Introduction}

Planning the spending of public funds is extremely important from the point of view of national strategy and should reflect the objectives of economic policy based on long-term development trends. Effective and efficient programming of support for research and innovation activities must be based on two assumptions: reliable and up-to-date data, and transparency of activities and independence from current political objectives. When implementing the strategic objectives of economic policy, one should bear in mind the separation from political pressures. In practice, this may be extremely difficult. It is worth repeating after Schumpeter [1] that "the typical citizen drops down to a lower level of mental performance as soon as he enters the political field". 
52 From the point of view of the organization in which intervention is planned, the transparency of the process and elimination of organizational pathologies, especially nepotism, require attention. A negative effect of nepotism is the "privatization" of some processes in the organization, where activities, including those resulting not only from the current functions of the system, but also planning and creating strategies are subject to the particular interests of small, informal and usually very hermetic interest groups. In order to reduce these negative phenomena, it is necessary to use clear and transparent procedures, but also tools (including information technologies (IT) tools). All this builds unique strategic knowledge. In commercial organizations, knowledge is difficult to copy by the competition, but for each organization it is a unique, strategic resource [2].

Support programming uses knowledge management instruments; both in the soft layer (human capital, social capital, cultural capital) and hard layer (databases, IT, etc.). It is important for the programming process to be based on systematic research of the environment and the accumulation of knowledge. Strategic change takes the form of an increase in the importance of organized research, the implementation of which is often supported by external institutions - entities from the science sector or specialized research and development (R\&D) entities. The role of interdisciplinary teams involving external experts is becoming increasingly important. Over time, these teams must acquire new competences in the use of advanced quantitative methods using IT [3]. Data-intensive methods based on IT tools are excellent complements to existing research and analytical methods. This is a way to discover potentially new relationships and improve theory [4]. Monitoring methods which are necessary for programming support for public funds must come out from the organization's mission. Knowledge management processes begin to define the identity and basic competencies of the organization. As with any other organization, those involved in the intervention process have their future depending on the environment, therefore it is necessary to answer the question, what are the dominant 
development trends. This question defines the scope and method of acquiring knowledge about the organization's environment, and consequently translates into the formulation of its strategic aims.

The issue of timeliness, availability and reliability of data, which were used in the programming as an essential decision-making process so far, is characterized by a relatively long delay to the phenomena or problem it described. For example, the feasibility studies for sectoral programs submitted in 2016 were most often based on statistical data preceding 2014 and for this reason did not fully reflected the actual condition and potential of the sectors. In addition, there is a lack of objective and up-to-date information on the current and planned activities of organizations implementing research and development.

Developing methods of data collection and analysis, including in particular information refining and Big Data analysis meet the needs of the programming process in terms of obtaining up-todate and objective information. The use of Big Data analysis allows for more effective monitoring of milieu of the economic sectors. The data obtained in the process of information refining are also independent of the direct influence of ad hoc political interests.

The method we describe is the first application of information refining and Big Data analysis in the programming process in Poland and is one of the first applications of these methods by public administration in the world.

New methods of monitoring the environment, especially those using artificial intelligence (AI), business intelligence systems, Big Data and information refining, are also reflected in the case of programming processes of $R \& D$ support [5] and begin to constitute an element of the knowledge management system. The efficiency of management systems depends on the ability to "create, transfer, pool, integrate and exploit knowledge resources" [6]. The new methods are therefore an assumption for a strategic change in the acquisition and analysis of data from the environment. However, it should be remembered that Big Data is not a panacea for all issues 
related to the analytics of large data sets. Big Data does not eliminate the need for intuition and creativity [7]. It is most important, whether the organization is doing enough to develop the

104 skills and competences to achieve the strategic aims. The affirmative answer is also an indicator

105 of the organization's ability to activate a powerful driver of competitive advantage [8].

106 New way of monitoring the milieu consisting of refining information method and Big Data

107 analysis was applied to the identification and exploration of network resources in search of

108 keywords and phrases on the construction materials technologies. The collected data were

109 cleansed and lemmatized and then analyzed with the use of Big Data tools. At this stage, data

110 were structured and subjected of the statistical analysis and then the desired information was

111 obtained.

\section{Case description}

114 Programming of support for R\&D activity should include purposeful operations based on the

115 principles of communication rationality. The measure must refer to an objective, social and

116 subjective world, it must also be characterized by normative rightness [9]. Such approach in the

117 programming process of support for R\&D activity consists, among others, in integrating the

118 scientific and business environment around the issues of support. It is also important to use

119 adequate, current and reliably processed data. There are many good practices scoping of

120 integration of scientific and business communities around common strategic objectives co-

121 defined by the administration. For instance, the integration of professional communities at the

122 level of voivodships was facilitated by work on regional strategies. In this case, the process of

123 work on strategies had a significant impact on the creation and development of relations and

124 improvement of competences. A good example of building cooperation and developing social

125 capital necessary for the creation of efficient institutions supporting R\&D was also the long-

126 term strategy of development in Poland. 
127 Monitoring a dynamically changing environment, especially in the field of $\mathrm{R} \& \mathrm{D}$, is a 128 particularly difficult task. Statistics covering R\&D issues face a number of problems which 129 make analyses not fully reliable, and reliable data may not fully reflect reality. This is due to

130 several reasons, some of which concern the issue of reporting to the Central Statistical Office 131 (GUS) and the reliability of data (both in terms of their scope and "quality"). Difficulties in 132 obtaining reliable data at the time when they are necessary concern many processes (including 133 the management of R\&D support activities). These include difficulties in comparing data both 134 internationally and interregionally, and significant delay in time (which often makes it 135 impossible to reliably compare economic data, influencing the programming process of 136 interventions) as well.

137 Monitoring of R\&D activity must go hand in hand with cyclical research of demand for 138 innovations. However, it remains an open question to what extent entrepreneurs should be 139 involved in the process of defining strategic aims of innovation support. This issue is 140 particularly important when we treat commercial companies only as "distributors" of 141 innovations which are "produced" by the science sector and should be introduced to the market.

142 Such a narrow understanding of the role of business, limiting the possibilities of using in 143 practice solutions such as open innovation or social capital, limit the potential effectiveness of 144 programmed support. On the other hand, the demand of commercial organizations for the 145 results of R\&D (in the future) is based on the current demand for products and services created 146 by such organizations. Planning of activities in a business sector is mostly based on 147 extrapolation of the present conditions (in the scope of both demand and the potential to satisfy

148 it). From the point of view of the R\&D support programming process, a greater integration of 149 the innovation process is necessary [10].

150 The complexity of the described processes concerns the growing wealth of data for analysis. 151 The analysis process itself is becoming more and more complex. The large amount of ambient 
data makes it difficult for the analytical process to focus on the need for optimal information

153 for the programming process. On the other hand, at each stage of the innovation process, the 154 organization generates information in various forms in virtual space (including, above all, the 155 Internet). From the point of view of monitoring the environment, an important task is to identify 156 this information, its refining and analysis. In order to effective R\&D support, it is therefore 157 necessary to identify and exploit data sources which, in a similar way, show data covering the 158 behavior of innovative entities at different stages of the innovation process. From this point of 159 view, it is also necessary to identify the processes of exchange of knowledge of innovative 160 organizations with the environment; when we know the way in which organizations search for 161 information which is necessary from the point of view of their processes, when we are able to 162 identify this information, then the effect of their analysis will bring knowledge about the 163 implemented and planned processes involving R\&D activity [11].

164 The exchange of knowledge, both within the company and with its environment, is influenced 165 by trust, values and norms. An important factor is the use of IT tools by the innovator. 166 Monitoring of sources, where the innovator leaves traces indicating both the technologies and 167 solutions he is looking for, as well as) the knowledge developed within the organization is a 168 key element of the modern programming process.

169 An example of a practical attempt to describe current and future trends important for innovation 170 processes is the use of Big Data and data refining within a joint project implemented by the 171 University of Warsaw and National Centre for Research and Development. The applied solution 172 may initiate a systemic change in the area of monitoring technological and scientific

173 environment. The tool enables advanced analysis of structured and unstructured data contained 174 in available databases (relational, hierarchical, network, object-oriented, etc.), file sets, portals, 175 websites and Internet forums, streaming transmissions and other sources on the basis of set-up 176 parameters (in particular: keywords and semantically similar: model images, model fragments 
177 of sound recordings, including speech recording, fields and scientific disciplines, including newly created multidisciplinary areas, etc.). The specific objective is to create an ecosystem of interactive scientific, scientific-technical and business information on technologies, based on the sequential analysis of structured and unstructured data available in distributed digital

181 repositories for science, education and an open knowledge society, of which analytical methods 182 and tools are an integral part:

- identification, on the basis of set parameters, of large data sources accessible via

The project includes data sources on R\&D and innovation processes contained in identified databases, unstructured sources, websites, social networking sites, forums, information portals, specialist sources of scientific and technical information, commercial and public data

194 collections, as well as the necessary methodologies for effective analysis of the acquired data.

195 The information obtained on the basis of information collected during the project 196 implementation is characterized by the following properties:

197 - is independent from the observer (objective),

198 - shows a synergy feature,

- is diverse,

- is an inexhaustible resource, 
- can be processed without causing wear and tear.

The aim of the article is to describe new ways of obtaining reliable and objective information for the strategic management of support for the national innovation system. This article presents partial results of a project on monitoring technology trends. The result of monitoring is not only the identification of technology trends and quantitative assessment of their dynamics of change and prediction.

The system created in the project makes it possible to distinguish strategic/dedicated sources of information for identifying technology trends. The following tools were developed:

- for automatic collection of source materials,

- for statistical processing,

- for quantitative analysis of historical, current and predictive data,

- $\quad$ systems for visualisation and description of results.

Procedures were defined for identifying dedicated sources and highlighting technology trends in selected areas of fields. The system includes the Analytical Warehouse containing dedicated source materials and software for feeding the warehouse with new data. The physical structure of the tool has a layered architecture, and the modular design ensures openness and scalability. The system is characterized by technological neutrality of the mutual information mechanisms and allows modernization of selected elements in the process of expansion. The data update of the Analytical Warehouse is provided by the Big Data Robot, a specialized IT system for targeted monitoring and collection of data from designated websites and offline sources, also in the form of digital text images, e.g. image documents, books or archived newspapers. The system consists of a number of simultaneously operating robots (agents), each of which, in specific and defined time units, interacts with a monitored information source - e.g. an information service. Selective retrieval software allows unnecessary information to be filtered out and a file prepared in a predetermined format to perform computations. It has a built-in 
function to convert text data saved in HTML format to plain text in UTF-8 format, and a

228 function to convert multi-line text to a single line with the removal of redundant spaces and 229 tabs. The selection of retrieved data reduces the randomness of collecting source material from 230 vast resources. The system ensures that source materials are integrated and standardized, 231 cleaned and classified, and collected in appropriate, thematic databases. The source material 232 databases, cleared of unnecessary characters, are the direct 'raw material' for performing 233 technology trend identification operations. Assigning materials to field-specific sections 234 (material dedication) and cleaning improve the efficiency and speed of technology trend 235 monitoring functions. These functionalities allow controlled integration of new material, 236 obtained from different sources, with previously collected resources, corresponding to the field 237 under study.

238 In identifying data sources, resource search strategies were defined and categorised. Strategies 239 are selectively applied depending on the research problem under analysis - the field, the 240 availability of sources and the information and search language used in the source. Specification 241 of tools for information retrieval has been developed, including traditional and specialized 242 search engines, scientific multi-search engines, search engines for documents saved in a specific 243 format. The available digital resources were systematized and the access points to information 244 from different sources were specified: social media aggregators, mashup tools, quality245 controlled catalogues, databases aggregating open scientific resources, and discovery systems. 246 The system is fed with resources related to innovations and technologies in the given field. A 247 mechanism for automatic document classification was developed based on machine learning 248 methods - supervised learning algorithms and natural language processing algorithms. This 249 mechanism will be used each time a new database is fed with new data to divide the database 250 resources according to the defined source categories. A mechanism was also created to classify 251 documents according to any classification, as an additional way of dividing database resources. 
252 The system allows for specification of technology trends concerning any area of the world and 253 enables analysis of the dynamics, directions and prediction of changes of identified threads as 254 well as cluster analysis.

255 The research uses tools to identify trends - elements of artificial intelligence, in particular 256 semantic analysis of Big Data using advanced methods of natural language processing. The 257 result of the analysis is a set of structured data characterizing each layer of the analysed text: 258 the token, syntactic group, sentence, section and the whole text.

259 The data refining method consists of cleaning and refining the information stored in large 260 information resources. Information created on the basis of data obtained in the refining process 261 will allow identification and analysis: quantitative description of the dynamics of changes in 262 technology trends in the dimensions of scientific information sources, administration and 263 business sources. The relevance of this analysis lies in the original method of analysing the 264 content related to the post (citation). Reflecting the content of the source materials containing 265 the posts are the post attributes - words occurring in the vicinity of the post. The quantitative 266 and qualitative description of the dynamics of changes in the frequency of occurrence of 267 attributes is an equivalent, with a similar description of the pole, part of the image of the pole. 268 In the adopted solution, attributes are exogenous variables.

269 The algorithm of the system for identifying technology trends is shown in Figures 1 and 2. 


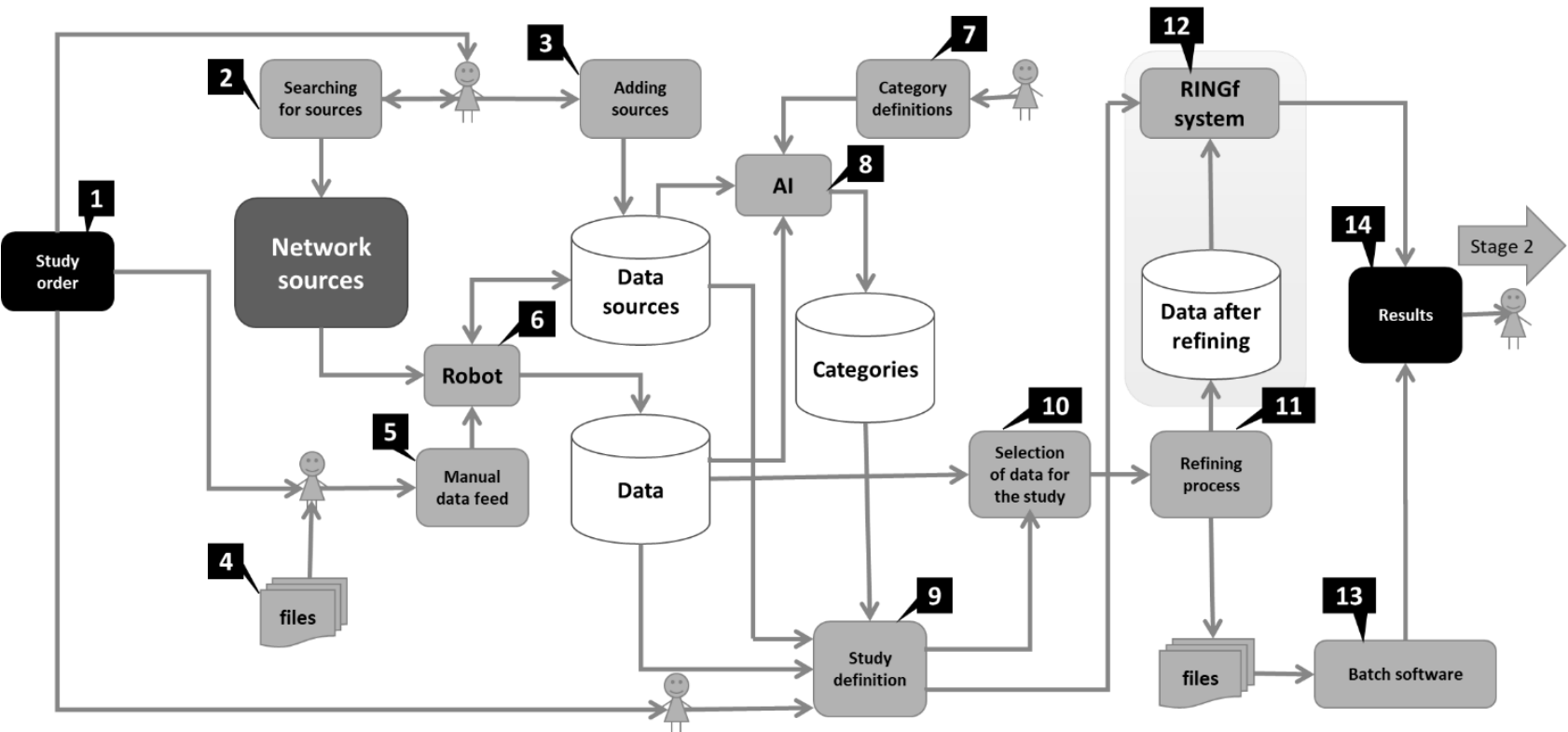

273 Figure 1: Algorithm of the system for identifying technology trends (own work)

Key:

1. Start - information necessary to determine the scope of the study. Includes, but is not limited to, the hypothesis, purpose, and assumptions. Due to the nature of the study, important input parameters determining, among other things, the assumptions are: the topic of the study, the time interval of the research, key words defining the subject of collection, performed analyses, etc.

2. Searching for sources - a process conducted by the researcher whose aim is to find appropriate sources of data according to the subject of the study. Searching for sources each time is done manually using the procedures described in this paper. At this stage, a division is made between sources that can be collected fully automatically by the robot and those that require semi-automatic or manual processing. 
3. Adding sources - actions performed from the administration panel, by means of which the researcher introduces sources of information to the source database. Based on the data entered, the robot proceeds to collect data. This process is fully automatic and no direct manual operation is required.

4. Files - represent any type of material that is not available in web sources. This includes the client's own material, which, for various reasons, cannot be accessed from the web. In a sense, we can refer to these materials as offline materials. These files include materials digitised by the researcher or the client as well as any other materials which require non-standard processing, e.g. the conversion of a multimedia file (image, audio) into text.

5. Manual data feeding - this is a functionality of the system that allows feeding data to the robot base, which the robot cannot handle automatically due to unavailability of this

6. Robot - a fully automated system for online data collection.

7. Category definition - based on the information provided in the order, the researcher defines categories of data for a given study. The defined categories are processed by AI algorithms in such a way that individual data sources are assigned to specific categories. The AI mechanism for category assignment is described in the Report.

8. AI - a separate fully automated AI engine built on top of TensorFlow.

9. Study definition - the activities that define a study in the system are derived from the order and processes described above. When defining a study, the researcher determines which data sources, which categories, and on which data the study will be conducted. The parameters defined in this section are the input configuration for the test data selection (10) and the refining process (11). 
314 10. Selection of data for the study - an automated process designed to identify specific 315 documents to be retrieved for the study - the refining process. The input data for the 316 selection are the boundary parameters given during the definition of the study based on 317 the order information.

318 11. Refining process - a fully automatic process implemented depending on the needs of 319 one or more computing nodes. As a result of the refining process, the system generates files with a database structure or saves data in the 'Data after refining' database.

12. Information Refining System (RINGf system) - fully autonomous system processing data after the refining process into human readable form. The data generated by the system in the form of raw results are the basis for analysis, on the basis of which reports are prepared in the second stage.

13. Batch software - includes proprietary software that performs data analysis after refining in an automated manner. Batch software, in addition to previously defined scenarios, can implement custom data processing algorithms.

14. Results - is a set of files that is the basic raw material needed to prepare the report. 


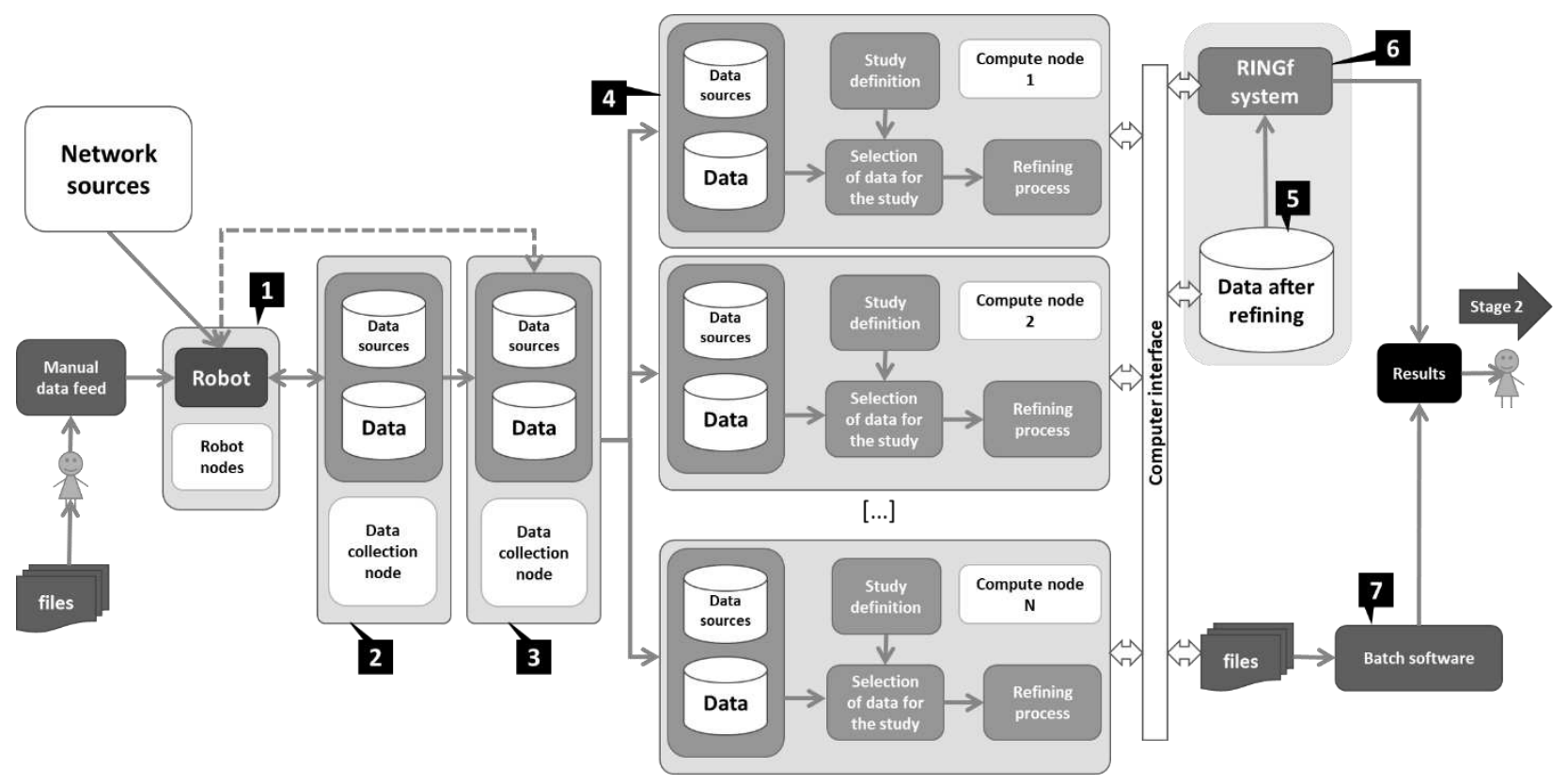

331 Figure 2: Data processing algorithm during identification of a technological trend (own

work)

Key:

1. The robot is a fully automated data collection system operating 24 hours a day.

2. It performs collection of data, which it stores in a database. The robot is run on several nodes in such a way as to ensure uninterrupted collection operation. Depending on the situation, the robot saved data in the primary database (2), and in case of failure or unavailability of the primary server, data is saved on the backup database (3).

3. Primary database server - This is the main server that accepts data. Its purpose is to save data to the database and perform replication to the backup server (3). No computation or information retrieval tasks are performed on the basic database server.

4. Backup database server - its main task is to accept a replica of data from the primary server and make the selected data available to servers acting as compute nodes. 
5. Compute nodes - these are powerful servers whose primary task is to perform computations. Each compute node has a replica of the database taken from a standby server. On servers, which are compute nodes, the tasks of data selection, data refinement processes, and computations are performed. More compute nodes can be added to the system as needed. On the compute nodes, computations are performed in parallel. The information broker is responsible for the division of tasks.

6. Data that are the product of refining are loaded into a dedicated database, which is used by the RINGf system or exported to files having a database structure.

7. RINGf system - is the previously mentioned fully autonomous system processing data after the refining process into human readable form.

8. Batch software - is the proprietary software described earlier for post-refining data analysis.

The modular design of the system ensures its open architecture and scalability, with respect to functionalities that are parametrizable depending on the subject of the study, the number of available information sources, the number of source materials. Scalability and openness also apply to the infrastructure of the system itself. This enables the use of distributed task processing. Processing is done in three compute nodes (strong servers, mainly thanks to superfast SSD/M2 drives) and 10 physical task machines.

The choice of a particular tool, resource or source is always dictated by the specifics of the research area under analysis (Figure 3). This, in turn, accounts for the fact that each search is

367 heuristic in nature and precludes the use of an invariant pattern that might apply to each search/identification of information sources conducted. The identification of information sources, through various tools (search engines, discovery services, databases, etc.), uses methods that relate to the theory and practice of using appropriate information search strategies. 
371 Search instructions are formulated that correspond to the assumptions of several strategies

372 (appropriate to a variety of situations, handling methods, but also sources characterized by a

373 different level of use of the controlled vocabulary). The application of specific search strategies

374 is always related to the research problem analysed, the availability of sources (e.g. specialist

375 sources), the available search tools, the information and search language used in a given source,

376 etc. Consequently, the same strategies are not used in every case of the analyses performed.

377 Depending on the various problems and research areas in which the aim is to identify trends 378 relating to the development of a specific phenomenon or technology, a different search and 379 identification strategy is established each time, as well as the use of different tool sets. The key 380 issues here are related to the given research area, the types of documents that the researcher will 381 process further, the applied method of collecting and exploring information (including 382 metadata), and above all, having appropriate knowledge and skills to use relevant information 383 resources adequately to the given situation.

384 This is complemented by tools provided by both, traditional and digital libraries or archives. 385 These tools provide one (though not the only) primary means of analysing technology trends 386 based on the current literature on a particular research problem and its accompanying metadata. 387 Importantly, this literature is not limited to academic papers (field know-how). Libraries today, especially academic and professional libraries, provide a number of tools that allow access to various types of documents, especially the so-called grey literature ${ }^{1}$.

\footnotetext{
${ }^{1}$ Anything that is not controlled by publishers, e.g., government, academic, industrial, business materials in print or electronic form, falls into this group of information resources. The latter group of documents includes scientific, technical, economic, social and other reports from public and private institutions, conference materials that are unpublished and not available through commercial publishing or bookshop chains, technical standards and recommendations, unpublished translations, articles published in short-run periodicals that are made available free of charge or of local circulation, certain official documents, technical, promotional and advertising documentation, and documents in electronic form.
} 
Discovery systems/multi-search engines enable rapid discovery and user-friendly delivery of

391 resources to which a library (or consortium of libraries) has access. These multi-search engines

392 offer integrated search of resources, including those belonging to the so-called hidden internet

393 (e.g. library catalogues, field-specific databases). The use of selected discovery systems makes

394 it possible to identify the situation in the research, development and innovation (R\&D\&I) area,

395 especially in the context of trend setting based on the most recent scientific literature. It also

396 allows a preliminary analysis of the phenomena in the environment and to indicate the

397 variability of this environment over time (in particular over particular years or specific

398 geographical areas). From the point of view of access to information on scientific publishing

399 production, the use of the WorldCat central catalogue also proves to be important. It should be

400 noted, however, that the catalogue itself is not a discovery system. However, due to the indexing

401 of information about the resources of libraries nearly the entire world (also in Poland), in this

402 case it is also worth noting the possibility of its use during the process of programming the

403 support for R\&D\&I activity. WorldCat allows narrowing search results using the Topic

404 category which is a kind of systematic arrangement in the form of a long drop-down list of

405 keywords having much in common with the classes of the information and search language of

406 the Library of Congress Classification [12].

407 In the course of the search and identification of sources, the law of Samuel C. Bradford (a

408 librarian of the Science Museum in London) is applied, according to which as many as 1/3 of

409 the publications concerning a given topic were published in works not belonging to a given

410 field. Publicly available, open and free sources of information (including open data) are

411 considered. In the case of Big Data mining, it is necessary to use an appropriate typology of

412 documents, which will enable proper organization of work related first to searching, organizing

413 and structuring of collected content, and then facilitate processing, data analysis and

414 visualization of the results of these activities. Data can come from a variety of sources, including 
unstructured sources - it is currently estimated that over $90 \%$ of information is stored precisely

416 in unstructured form [13]. For the purpose of source identification, two typologies of documents

417 are used: the classic, which refers to the traditional division of documents into primary,

418 secondary and derivative, and the typology strictly related to digital resources. Both typologies

419 provide a starting point for collecting data from various sources (also from the point of view of

420 Internet domains - .edu, .gov, .com or .org).

421 All content about systems, processes and events in the economy, but from different sources, is 422 considered. They, therefore, include both standard and specialized (e.g. scientific) search 423 engines, data from public institutions (e.g. government, local government, European Union), 424 commercial organizations, bibliographic (abstract) and full-text databases, patent databases, 425 social networking sites, blogs, microblogs and discussion forums, RSS feeds. This group also 426 includes data and documents presented in an open way, library catalogues and discovery 427 systems, thematic and business catalogues, databases about companies, content included in 428 information portals.

429 Data are collected from a wide variety of data sources, mostly available via the Internet. In 430 particular, Open Access: data from public institutions, government, commercial organizations, 431 social media, patent databases, grey literature, information portals, media, commercial 432 organizations, open data and others. In the majority, unstructured data are collected.

433 Access to publications is accomplished in two ways. The first, the so-called 'gold route,' 434 involves publishing in peer-reviewed journals. The second, so-called 'green way' is for 435 researchers to make their publications, including non-peer-reviewed publications, available in 436 open digital repositories and libraries (self-archiving). For the past decade or so, both the 437 number of journals and repositories have been growing rapidly. In the case of journals, these 438 are obviously new periodicals, but also existing ones that have changed their publishing model 439 to Open Access. Suber observes that an increasing number of these titles "are gaining a 
reputation as professional, prestigious periodicals of great scientific importance [...] There is

441 also a growing awareness that supporting OA journals is an effective way to support science,

442 researchers, scientific institutions, and the review process. More and more publishers of 443 journals that have become open access journals are noticing that their citation rates have 444 increased, as well as the number of papers retrieved" [14]. Open access journals, digital libraries 445 and repositories are being introduced by the best universities in the world (e.g. Harvard, MIT 446 or Princeton), which is directly related to the promotion of science, but also to strengthening its 447 role in the economic development of countries.

448 Given the crucial importance of the reliability of the source materials used in the system for 449 identifying technology trends, empirical research was conducted to verify the reliability of open 450 data. To this end - a comparison was made between the results of analyses stemming from the 451 documents coming from publicly available (open) sources and those stemming from the 452 analyses of licensed (closed) sources.

453 In order to feed the Analytical Warehouse with data related to innovation and technology, 454 resources were identified and entered into the database. This facilitated the start of the process 455 of automatic learning and assignment of data sources concerning a given field to specific 456 categories: business, science, administration, patents, tenders, media and communication, and 457 Web 2.0.

458 Big Data analysis is based on data in the form of plain text encoded in UTF-8 standard. In this 459 form, they are stored in the repository. Data must be converted to this form if it exists in another 460 format. Mechanisms have been developed for conversion from PDF (most scientific 461 publications are downloaded in this format), DOC, DOCX and ODT (a small number of 462 scientific publications), HTML (e.g. patents on Google Patents platform) and RSS (news feeds 463 of websites). In the case of PDF format, a small part of the source data requires an additional 464 operation to extract text from the image using OCR technology. In the following, the file 
formats mentioned above are referred to as non-text files. Input information is provided

466 primarily in the form of URL links directing to web pages containing data (e.g., results of searches from Google Patents), web pages containing links to non-text files with data (e.g., scientific journals, results of DOAJ repository searches), direct links to non-text files, RSS feed 469 addresses.

470 For the most part, neural networks are used to identify the values of metadata that constitute the 471 logical structure of the analytical warehouse, e.g. for: source classification, document 472 classification into the given field, publication language recognition. A different approach was 473 used for each of the above cases. Document classification into the given field - since in each 474 task a different field will be given, the model must be built on the basis of provided descriptions. 475 In this case, we will rely on the corpus and the neural network provided by the model 476 manufacturer Google (BERT model - the latest model developed for natural language 477 processing tasks). Publication language recognition - a model developed by Google was used 478 for publication language recognition. It is based on neural networks. In this project, we will not 479 build a neural network model, we will use the networks provided with the model. An automatic 480 data classifier was created as part of the system. Supervised learning algorithms and natural 481 language processing algorithms were used for this purpose. After data preparation, a division 482 was made between a learning set and a test set. The learning set contains the data on which the 483 machine learning algorithms train the model, and the test set is the set left to confirm the 484 classification results of the learned model. The test set is not used during training because as a 485 rule it should be invisible for the model so that the test results are reliable. The processed input 486 of the learning set was fed to the deep network, which learned its internal dependency 487 representations and created a classification model. The model thus created was used to predict 488 labels for a test set that underwent an identical transformation with the fastText algorithm. The 
prediction result for each record of the test set is a probability vector at the position

490 corresponding to the label position in the list.

491 One of the most important considerations when using tools that automate the process of 492 downloading data from the Internet is defining an appropriate value for the 'User Agent' 493 parameter. This parameter is a kind of 'business card' of a web browser. Thanks to this value, 494 the server learns which browser the request comes from. Some servers do not allow an empty 495 'User Agent' value or vary the response content depending on the value of this parameter. 496 Therefore, care must be taken to ensure that each of the tools used for data acquisition allows 497 it to be defined. When retrieving data for Big Data analysis, the 'User Agent' parameter is set 498 to identify the current version of the Chrome browser (e.g., 'Mozilla/5.0 (Windows NT 10.0;

499 Win64; x64) AppleWebKit/537.36 (KHTML, like Gecko) Chrome/60.0.3112.113 500 Safari/537.36').

502 The database constituting the Analytical Warehouse accepts sources in the form of RSS feeds, 503 which are then regularly checked for new messages, which poses a problem when feeding the 504 database with text document files. To solve the problem, proprietary software was created that 505 creates 'artificial' RSS feeds, also referred to as quasi-RSS, from downloaded documents. The 506 content of an RSS feed includes a list of 'messages', each with a date associated with the file 507 and a link leading to the text form of the document located on the server. An RSS feed prepared 508 in this way can be added to a database, where it will be processed in the same way as other 509 standard RSS feeds.

510 The process of creating quasi-RSS files is performed by a custom tool written in Perl called 511 make_quasi_rss. The input is a tree structure of directories containing non-text files, created by 512 the doaj_fetch or doaj_fetch_batch tool, or any directory containing non-text files at any nesting 513 level. The program performs data extraction, converts the non-text file to text, saves the 
514 resulting text in a UTF-8 encoded text file under a unique name obtained from calculating the

515 sha256 checksum of the input non-text file. On the basis of the obtained text files, an XML file 516 compliant with the RSS specification is created containing links to the created text files. The

517 program saves information in the database such as the original file name, the sha256 checksum, 518 the date from the metadata, information about whether the file requires an OCR process and 519 whether the conversion proceeded without error.

521 The database used to conduct the research includes sources from many different services and 522 appearing in different formats. Therefore, before starting computations for each study, the 523 database must be checked for completeness of the sources it contains dedicated to the study that 524 were previously added. Depending on the type of source, the verification method may vary. A 525 quantitative method of comparing the number of sources downloaded by proprietary software 526 to the number of sources in the database. In case of a difference of more than $10 \%$, the process 527 of downloading and adding sources to the database is repeated. The method consists in 528 comparing individual items from the list of sources prepared for the study with the sources 529 present in the database. If any source is missing, it is added again to the database until the match 530 between the source list and the database is $100 \%$. 


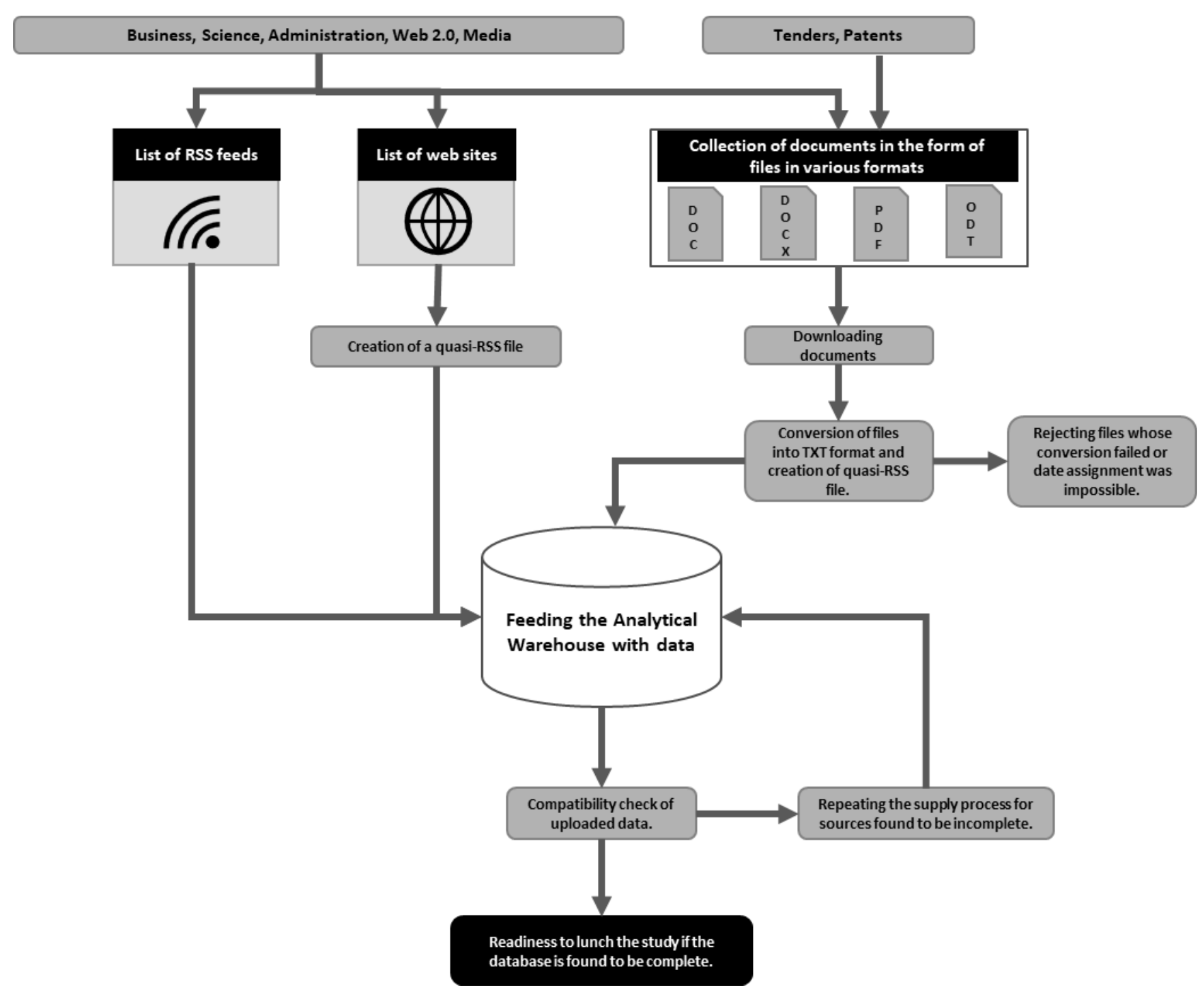

533 Figure 3: The process of feeding information to the system for monitoring technology

\section{4 trends (own work)}

535 The analytical tools applied in the system for identifying technology trends are used in the

536 following sequence of operations: tokenisation; quantitative analysis; TF-IDF statistics; bigram

537 analysis; correlation analysis; cluster analysis.

538 The search and identification of technology trends takes place on the basis of source materials

539 belonging to the field indicated. The classification of industries is intended to define the area to

540 which the document applies. Industry classification is made at the document level. Not every

541 document can be clearly classified into the right category. Documents that cannot be assigned 
to a category with the appropriate confidence level cannot be mapped. The confidence level

543 should be determined by the machine learning model based on the statistic denoting the

544 similarity of text classification. The source documents should be automatically classified into

545 topic groups using machine learning methods. The machine learning model performs the

546 transformation of the category descriptions into numerical form. The same operation is applied

547 to the texts of publications and the measure of similarity is determined. A sufficiently high

548 similarity between the class and the text allows text classification. Due to the large number of

549 documents to be assigned, the classical supervised method of labelling a sample of articles for

550 each code (class) and using a machine learning algorithm would require a huge amount of effort

551 to collect and manually assign the learning material, which would not at all guarantee a good

552 classification result. Therefore, an unsupervised method based on the ready-made BERT

553 language model distributed in open-source mode by Google was chosen for the task. BERT is

554 the latest model developed for natural language processing tasks, which is based on the

555 architecture of deep neural networks learned on massive text sets. BERT stores representations

556 of words in over 100 languages as tensors in a multidimensional space and allows automatic

557 selection of the appropriate representation depending; on the context of the word. Performance

558 on standard language tasks places the BERT model at the top of the group of ready-made

559 models. The advantage of BERT over other models is the ability to automatically create tensors

560 for whole sentences and texts in different languages. Thus, texts of different lengths can be

561 compared for similarity. In order to assign a PKD code (Polish Classification of Activity) to

562 texts, each code description was first transformed into a 768-dimensional tensor using the

563 BERT model. The resulting tensors were saved in HDF format for quick access in the next step.

564 The code transformation is required only once, and the finished tensors created on both

565 languages can be used automatically in new iterations on new data. After converting the codes,

566 each English text was also converted to tensors using the BERT model with the same 
parameters as the PKD codes. After converting the texts, the tensors were used to calculate a similarity measure between the text and the PKD codes. The distance in Euclidean space, which is used in this type of cases, was used as a measure. For each text tensor, a similarity measure was created with the PKD code. The most similar PKD tensor was selected and its code was finally assigned to the text. In addition to the code, a distance measure in normalized form 572 (range 0-1) was also assigned to each text. The entire operation was performed automatically with the FAISS library, which was made available on an open-source basis by Facebook. FAISS is used for express computation of vector similarity on a large amount of data, which allows very efficient computation compared to classical methods for computing distances in multidimensional space. Analogically, for the assignment of PKD codes in English, tests for

577 Polish were carried out using the BERT method. After analysing the results, it was found that 578 the algorithm did not perform very well due to the accidence of the Polish language. The verbal forms that occurred in the texts did not translate into the forms found in the PKD codes. One could try to solve the problem by reducing all words to their basic form (lemmatisation); however, there is no open model that would be able to perform this operation in a satisfactory

582 time on the Polish language. For this reason, it was decided to use another unsupervised method, 583 namely the fuzzy matching method.

584 In order to obtain the greatest possible substantive value of the results of research implemented in the system for identifying technology trends, it was necessary to launch a procedure 586 classifying materials for a given field according to the criteria of category. Source classification 587 is done to recognize where a document comes from to be able to use the specifics of the source. 588 The problem is to seek differentiated assessments of identified technology trends. For example, 589 the categories business, tenders, patents are a form of indication of the intensity of technology 590 consumption while science assesses the dynamics and prediction of technology change. 591 Documents from social networking sites may be interpreted differently than scientific or patent 
publications for research on technology trends. In addition, it is possible to identify differences

593 in the popularity of technology between information from the business and academic worlds

594 based on data from different categories.

595 In order to create a mechanism for automatic publication categorisation, the learning set was

596 classified. Each record was manually assigned by experts to one of seven thematic groups.

597 The R scripting language was used to create an automatic data classifier using supervised

598 learning and natural language processing algorithms. The input data was developed in an Excel

599 spreadsheet containing records divided into columns, and the result of the script is a finished

600 model and measures of its effectiveness.

601 Publications that were not ranked by experts were ranked using a machine learning model.

602 Supervised learning and natural language processing algorithms were used to create an

603 automatic data classifier. The input data were unclassified records representing data sources.

604 The result was a finished model and measures of its effectiveness.

605 Model verification consisted of comparing the category prediction made by the model with the

606 classification made by the experts. For this purpose, a test set was used which the learning

607 model is not familiar with. A confusion matrix was created to compare the categories given by

608 the model with the human-made categorisation. The whole model accuracy - the number of

609 correctly performed categorisations for all test cases is $0.84 \%$. The trained machine learning

610 model was used to map the remaining sources - not assigned by experts.

\section{Discussion and Evaluation}

612 In collecting, storing and analysing data, the presented system uses elements of artificial 613 intelligence, in particular semantic Big Data analysis using advanced natural language 614 processing methods. The applied data refinement method includes cleaning, lemmatisation and 615 refinement of data collected in identified and clustered big information resources. 
616 The purpose of the tool is not only to identify trends relating to the development of a particular

617 phenomenon or technology, but also to precisely define the search strategy itself and to identify

618 and use a particular set of analytical tools. To this end, the tools developed are based on

619 advanced artificial intelligence solutions. In the course of testing and using the tool presented

620 here, several key issues were identified and diagnosed that may hinder wider use of the

621 presented solution. Among other things, lack of, or insufficient knowledge and ability to use

622 relevant information resources adequately for a given situation was identified as a key barrier.

623 All content about systems, processes and events in the economy, but from different sources, is

624 considered. Familiarity with the processes themselves can also be a significant barrier to

625 effective use of the tool by employees of public institutions.

626 In addition, the procedure that classifies materials for a given discipline according to categorical

627 criteria can be a significant limitation - changing (external) categorisation criteria and

628 catalogues, which is the result of changes in science policy, can greatly limit the formal

629 applicability of the results of the analyses conducted.

630 However, potential problems or risks associated with the management of the process of analysis

631 and use of information from the tool do not change the fact that the solution has great potential

632 for implementation. This solution allows for a more effective science policy to be pursued by

633 the institutions called upon to do so.

634 The model verification showed that, in the comparison of the predictions made by the described

635 tools with the classifications made by experts, the former are more accurate and precise.

636 Moreover, the results of the analyses performed by the presented model are not influenced by

637 distorting factors - fads, trends, political pressures, or processes with unidentified, non-

638 substantive background. It should be emphasized that the accuracy of the whole model is 0.84 .

639 Over time, this relevance increases as the applied machine learning model using artificial

640 intelligence algorithms learns during successive iterations of the analytical process. 
641 As part of tool testing, a test identification of innovation processes and monitoring of selected

642 technology trends was carried out. Test results suggest that the tool presented here will

643 contribute to a high degree to a more efficient allocation of resources within the framework of

644 support instruments used by public institutions.

\section{Conclusions}

646 The actual technological and scientific potential of beneficiaries of R\&D support programmes

647 can be determined with methods going far beyond the existing research and analytical tools 648 used by public institutions.

649 Modern methods of identification and diagnosis of technology trends, presented in the article, 650 allow for better adjustment of the support offered for R\&D activity, but also make it possible 651 to apply and improve management methods in public institutions.

652 The system for identifying technology trends presented in this article is one proposal for a 653 solution which enables more effective management of public funds directed for the 654 development of innovative activity. The presented solution makes it possible for public funds 655 at the disposal of support institutions to be spent in a way which will be better adjusted not only 656 to the needs of scientific institutions and enterprises carrying out R\&D activity, but also to the 657 scientific and technical potential of these organizations.

658 The tools presented make it possible not only to distinguish strategic sources of information for 659 identifying technology trends, but above all to automatically collect and process unstructured 660 data. The tools allow for quantitative analysis of this data, prediction in defined areas and 661 visualization of the results of analysis.

662

663 List of abbreviations

664 AI - artificial intelligence

665 GUS - Statistics Poland (Polish national statistical office) 
IT - information technologies

667 PKD code - Polish Classification of Activity code

668 R\&D - research and development

669 R\&D\&I - research, development and innovation

670 RINGf system - Information Refining System

671

672 Declarations

673 Ethics approval - not applicable

674 Consent to participate - not applicable

675 Availability of data and material - in the text

676 Competing interests - not applicable

677 Funding - National Center for Research and Development

678 (Cybersecident/489281/IV/NCBR/2021), University of Warsaw

679 Authors' contributions - authors' contributions are equal.

680 Acknowledgements

681 The authors thank the National Center for Research and Development for funding the project

682 and the Faculty of Political Science and International Studies for supporting its implementation.

683

684 References

685 1. Schumpeter J. A. (2003), Capitalism, Socialism and Democracy, George Allen\&Unwin 686 (Publishers) Ltd, 1976, Edition published in the Taylor \& Francise-Library

687 2. Żołnierski A. (2015), Nieformalne źródła informacji w działalności gospodarczej, in: W.Cetera, K.Kowalik (ed.), Logistyka $i$ administrowanie $w$ mediach, Instytut Dziennikarstwa Uniwersytetu Warszawskiego

690 Available from: Logistyka mediów - 2014 mono.pdf (uw.edu.pl) 
3. Ohlhorst F. J. (2015), Big Data Analytics: Turning Big Data into Big Money, John Wiley\&Sons, Inc.

4. Chai S., Shih W. (2017), Why Big Data Isn't Enough, "MIT Sloan Management Review”, Winter, 58(2) Available from: Chai, Shih - 2017 - Why Big Data Isn't Enough | PDF | Mathematical Model $\mid$ Weather Forecasting (scribd.com)

5. Richards G. (2018), Big Data and Analytics Applications in Government: Current Practices and Future Opportunities, CRC Press Available from: Big Data and Analytics Applications in Government: Current Practices $\underline{\mathrm{a}(\text { routledge.com) }}$

6. Frishammar J., Richtnér A., (2008), Editorial, "Int. J. of Technology Intelligence and Planning", 4:(3) Available from: International Journal of Technology Intelligence and Planning (IJTIP) $\underline{\text { Inderscience Publishers - linking academia, business and industry through research }}$

7. Hayashi A. M. (2014), Thriving in a Big Data World, "MIT Sloan Management Review", Winter, 55(2) Available from: Winter 2014 (mit.edu)

8. Ben-Hur Sh., Jaworski B., Gray D. (2015), Aligning Corporate Learning With Strategy, MIT Sloan Management Review”, Fall, 57(1) Available from: Aligning Corporate Learning With Strategy - MIT SMR Store Massachusetts 
10. Moor K. De, Berte K., Marez L. De, Joseph W., Deryckere T., Martens L. (2010), Userdriven innovation? Challenges of user involvement in future technology analysis, “Science and Public Policy”, February, 37(1) Available from: (6) (PDF) User-driven innovation? Challenges of user involvement in future technology analysis (researchgate.net)

11. Stephens-Davidowitz S. (2017), Everybody Lies: Big Data, New Data, and What the Internet Can Tell Us About Who We Really Are, Dey

12. Hollender H., Deskryptory i przyszłość opracowania rzeczowego w bibliotekach, „Rocznik Biblioteki Narodowej” 2015, R. 46, s. 406

13. Gang-Hoon, K., Trimi, S., Ji-Hyong, C., Big-Data Applications in the Government Sector, „Communications Of The ACM”, 2014, vol. 57, no. 3, s. 78 Available from: (6) (PDF) Big Data Applications in the Government Sector: A Comparative Analysis among Leading Countries (researchgate.net) Available from: www.ebib.pl/images/stories/numery/125/125_suber.pdf 